\title{
El aprendizaje colaborativo: Bases teóricas y estrategias aplicables en la enseñanza universitaria
}

\author{
Collaborative learning: Theoretical foundations and \\ applicable strategies to university teaching
}

\author{
Nestor D. Roselli ${ }^{12 a}$ \\ 'Pontificia Universidad Católica Argentina, Ciudad Autónoma de Buenos Aires, \\ Argentina. \\ ${ }^{2}$ Centro de Investigación en Psicología y Psicopedagogía, Ciudad Autónoma \\ de Buenos Aires, Argentina.
}

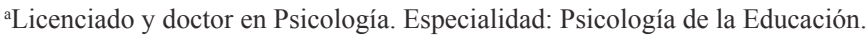
Investigador principal del CONICET. Director del Centro de Investigación en Psicología y Psicopedagogía de la Pontificia Universidad Católica Argentina.

Recibido: 07-10-15

Aprobado: 05-01-16

\section{Correspondencia}

Email: nestorroselli@uca.edu.ar

\section{Citar como:}

Roselli, N. (2016). El aprendizaje colaborativo: Bases teóricas y estrategias aplicables en la enseñanza universitaria. Propósitos y Representaciones, 4(1), 219-280. doi: http:// dx.doi.org/10.20511/pyr2016.v4n1.90

\section{Notas}

La presente investigación contó con el financiamiento de la Agencia Nacional de Promoción Científica y Tecnológica (Argentina), PICT 0960

(C) Universidad San Ignacio de Loyola, Vicerrectorado de Investigación y Desarrollo, 2016.

(๕) BY-NC-ND Este artículo se distribuye bajo licencia CC BY-NC-ND 4.0 Internacional (http://creativecommons.org/licenses/by-nc-nd/4.0/). 


\section{Resumen}

El Aprendizaje Colaborativo es un constructo que identifica un campo de fuerte actualidad, tanto en educación presencial como virtual. En primer lugar, se analizan las tres fuentes teóricas convergentes: la teoría del conflicto sociocognitivo, la teoría de la intersubjetividad y la teoría de la cognición distribuida. En segundo lugar, se presenta un modelo propio de estrategias que el docente puede implementar para desarrollar la colaboración sociocognitiva. Dicho modelo integra y sistematiza diversas técnicas de animación grupal con fines académicos desarrolladas dentro del campo del Aprendizaje Colaborativo. Precisamente es esta integración, dentro de una intención didáctica coherente y unificada, la que habilita hablar de estrategias más que de técnicas sueltas y disociadas. Se describe concretamente cada una de las estrategias, las que se refieren a seis ejes: Estímulo del diálogo, la escucha del otro y la evaluación recíproca; Colaboración para la negociación y creación de consenso; Organización de la actividad; Estudio y apropiación de la información bibliográfica; Elaboración conceptual; Escritura colectiva. Las estrategias propuestas (pensadas para estimular la colaboración entre dos, tres, cuatro y, excepcionalmente, seis u ocho estudiantes) no son las únicas posibles; se pueden combinar o asociarse con variaciones que el propio docente puede crear. Una característica de la propuesta es la estricta pautación de cada estrategia. También se alienta al docente a evaluar comparativamente los logros de aprendizaje obtenidos con cada estrategia con los obtenidos con modalidades individuales o no colaborativas. Finalmente, se exponen conclusiones y recomendaciones para la implementación de dichas estrategias.

Palabras clave: Aprendizaje colaborativo, aprendizaje cooperativo, enseñanza, constructivismo, trabajo en equipo. 


\section{Summary}

Collaborative learning is a construct that identifies a current strong field, both in face-to-face and virtual education. Firstly, three converging theoretical sources are analyzed: socio-cognitive conflict theory, intersubjectivity theory and distributed cognition theory. Secondly, a model of strategies that can be implemented by teachers to develop socio-cognitive collaboration is presented. This model integrates and systematizes several academic group animation techniques developed within the collaborative learning field. These integrated techniques, within a coherent and unified didactic intention, allow talking more about strategies than independent and dissociated techniques. Each strategy is specifically described, which refers to six areas: encouragement of dialogue, listening to others and reciprocal assessment; collaboration for negotiation and consensus building; activity organization; study and appropriation of bibliographic information; conceptual development; collective writing. These strategies proposed (designed to stimulate the collaboration between 2, 4 and exceptionally, 6 or 8 students) are not the only possible strategies, they can be combined with the ones the teacher might suggest. The strict pattern of each strategy is a characteristic of the proposal. The teacher is also encouraged to benchmark the results obtained using each strategy and those obtained using individual or noncollaborative strategies. Finally, conclusions and recommendations for the implementation of these strategies are discussed.

Keywords: Collaborative learning, cooperative learning, teaching, constructivism, team work. 


\section{Introducción}

\section{Objetivos.}

El presente trabajo tiene dos objetivos básicos: uno esencialmente teórico y otro eminentemente práctico. En ambos aspectos, la bibliografía existente sobre el aprendizaje colaborativo presenta problemas de sistematización e integración de toda la información disponible.

En lo que hace al aspecto teórico, se hace necesario acercar comparativamente las diversas fuentes conceptuales convergentes. Este es el primer objetivo del trabajo. En tal sentido se analizan tres grandes teorías fundantes: la teoría del conflicto sociocognitivo, la teoría de la intersubjetividad y la teoría de la cognición distribuida, haciendo además referencia al aprendizaje colaborativo mediado por computadora y a la corriente de la dinámica de grupo.

En lo que hace al aspecto práctico, el trabajo propone un modelo integrado de estrategias (más adelante se explicará la razón del uso de este término, más que el de técnicas) que el docente puede implementar dentro de una concepción didáctica colaborativa. Se habla de modelo porque no se trata de meras técnicas disociadas, sino de formas de operar que apuntan todas al desarrollo de la colaboración en torno a los grandes segmentos didácticos o ejes de toda didáctica colaborativa: Estímulo del diálogo, la escucha del otro y la evaluación recíproca; Colaboración para la negociación y creación de consenso; Organización de la actividad; Estudio y apropiación de la información bibliográfica; Elaboración conceptual; Escritura colectiva. Por lo dicho, tales estrategias no están pensadas como técnicas sueltas, sino formando parte de una unidad teórico-práctica que abarca todo el hecho didáctico. El aprendizaje colaborativo auténtico implica una comunión ideológica y operacional; y, en este sentido, ambos objetivos son convergentes. 


\section{Fundamentos teóricos.}

El aprendizaje colaborativo es un concepto que define un área teórica y de investigación de gran actualidad y de fuerte identidad. Aunque el tema de la cooperación intelectual tiene una larga tradición en el ámbito de la investigación en psicología y educación (Melero Zabal \& Fernández Berrocal, 1995; Roselli, 1999a; Rodriguez Barreiro, Fernández, Escudero \& Sabirón, 2000; Barkley, Croos \& Major, 2007; Strijbos \& Fischer, 2007), muchas veces asociado a la idea de trabajo en grupo o en equipo, recién en la década de los 80, y sobre todo de los 90, la cuestión cobra un nuevo impulso, dando lugar al campo epistémico reconocido como aprendizaje colaborativo.

De hecho, en esta nueva versión de la coparticipación cognitiva, el término "colaboración" desplazó al más tradicional "cooperación". En este sentido, si bien no hay un criterio unívoco, e incluso se los llega a usar de modo indistinto, se suele establecer una diferencia entre ambos (Dillenbourg, 1999; Dillenbourg, Baker, Blaye \& O’Malley, 1996; Lewis, 2003; Panitz, 1997). Existe un cierto consenso que define a la cooperación como una división de funciones basada en una repartición de la tarea, lo cual daría lugar a un segundo momento de ensamblaje grupal. La colaboración sería, en cambio, un proceso colectivo desde el inicio, donde todos intervienen conjuntamente en la realización de la tarea. Esto no implica que no pueda haber una natural diferenciación de roles, pero esta es una emergencia espontánea de la dinámica interactiva. En los términos de Dillenbourg (1999), se trataría de una diferenciación horizontal, y no vertical, como sería el caso de la cooperación.

Pero la diferencia es más profunda que la referida, como lo reconocen Barkley et al. (2007). Se trata de una diferencia de las bases epistemológicas. La línea de investigación sobre cooperación es básicamente anglosajona; los hermanos Johnson y Slavin son sus representantes más conspicuos (los títulos 
más representativos en castellano son: Johnson \& Johnson, 1999; Slavin, 1999; Huertas, 2001, que, aunque es catedrático en la UA Madrid, realiza una síntesis de estos autores). En esta corriente, el peso de la enseñanza recae en el docente, quien detenta el conocimiento que debe ser aprendido por los alumnos. El trabajo cooperativo consiste en la aplicación, por parte del docente, de técnicas grupales dirigidas a lograr este objetivo; en tal sentido, su uso es instrumental y complementario. La cooperación no es una ideología generalizada de toda la enseñanza; es una parte del proceso, donde se recurre a la cooperación entre pares como una manera de afianzar los logros de aprendizaje. Estas técnicas encuentran su espacio ideal en la educación primaria y secundaria. No están pensadas para la educación superior, donde la población es adulta y el conocimiento es de una alta especialización. Con todo, en los últimos tiempos han aparecido en castellano manuales referidos al uso de técnicas colaborativas en la universidad (Barkley et al., 2007; Exley \& Dennick, 2007), que incluso apelan a este nombre, pero la impronta funcionalista denuncia su raíz anglosajona.

Por el contrario, el enfoque del aprendizaje colaborativo se inscribe dentro de una epistemología socioconstructivista (Bruffee, 1993) o, empleando las palabras de Quiamzade, Mugny y Butera (2013), de una "psicología social del conocimiento". El conocimiento es definido como un proceso de negociación o construcción conjunta de significados, y esto vale para todo el proceso de enseñanza-aprendizaje. Aunque el peso del concepto está puesto en el reconocimiento del valor de la interacción cognitiva entre pares, el aprendizaje colaborativo involucra también al docente y, en general, a todo el contexto de la enseñanza. No se trata, pues, de la aplicación circunstancial de técnicas grupales, sino de promover el intercambio y la participación de todos en la construcción de una cognición compartida.

Las raíces teóricas del aprendizaje colaborativo, de inspiración neo-piagetiana y neo-vygotskiana, son bastante diferentes de la línea del aprendizaje cooperativo, más cerca de la corriente de los "pequeños grupos" 
y de las habilidades sociales. En la perspectiva "colaboracionista" convergen tres teorías de significativa vigencia: la teoría del conflicto sociocognitivo, la teoría de la intersubjetividad y la teoría de la cognición distribuida (Roselli, 2007), las tres expresión del auge socioconstructivista en la psicología y la educación.

\section{La teoría del conflicto sociocognitivo.}

La teoría del conflicto sociocognitivo se inscribe en lo que la Escuela de Psicología Social de Ginebra, responsable de la sistematización de esta, llamó "paradigma interaccionista de la inteligencia". Esta postura debe entenderse en el contexto del pensamiento piagetiano, como una derivación crítica de este. Por eso se la puede caratular de neo-piagetismo, a pesar de que la importancia que sus representantes le asignan a la interacción sociocognitiva los acerca a la perspectiva vygotskiana. De hecho, puede considerársela un enfoque socioconstructivista (Dillenbourg et al., 1996).

Para esta teoría, el conflicto sociocognitivo constituye el factor determinante del desarrollo intelectual. Este se vehiculiza en el seno de la interacción social, fundamentalmente en contextos de cooperación entre pares. La multiplicidad de perspectivas que convergen en este tipo de situaciones sociales, siempre que sean intrínsecamente conflictivas y que den lugar a un desacuerdo social explícito, hace posible la descentración cognitiva del sujeto y, con ello, el progreso intelectual.

El concepto de conflicto cognitivo está implícito en la teoría de la equilibración, frecuentemente entendido como perturbación del equilibrio en la relación sujeto-objeto. Como dice Coll (1991): "En lo que podríamos llamar la versión ortodoxa piagetiana, el conflicto cognitivo aparece básicamente como resultado de la falta de acuerdo entre los esquemas de asimilación del sujeto y la constatación de los observables físicos correspondientes, o bien 
como resultado de las contradicciones internas entre los diferentes esquemas del sujeto" (p. 116).

Para la Escuela de Psicología Social de Ginebra, esta es una idea individualista del desarrollo. El conflicto cognitivo que posibilita el progreso operacional es ante todo social, o sea que ocurre en una situación de intercambio (cooperativo) con otros. Antes de ser individual, el conflicto es social. Es gracias a este que el sujeto puede superar el egocentrismo cognitivo (centración en sus esquemas propios preexistentes). Solo a través del conocimiento de las perspectivas ajenas, el sujeto puede modificar sus propios esquemas. No se trata de un conocimiento estático, sino de una negociación activa con el(los) otro(s) para llegar a algún tipo de consenso.

\section{La teoría de la intersubjetividad.}

Para Vigotsky, lo mismo que para G. Mead, los procesos interpsicológicos preceden genéticamente a los procesos intrapsicológicos. Esto implica que la conciencia individual emerge gracias y a través de la interacción comunicativa con los otros. Lo importante de esta interactividad social primaria es que a través de ella se "internalizan" los instrumentos y los signos de la cultura. La mediación semiótica o cultural es fundamental en toda actividad humana, ya sea dirigida al mundo físico o al mundo social. Se entiende entonces por qué, para esta corriente, la interacción con los demás (y la interacción del sujeto consigo mismo) es básicamente dialógica, ya que se trata de una interactividad mediada por el lenguaje y otros sistemas simbólicos. La conciencia (como fenómeno intrapsicológico) emerge, pues, de la intersubjetividad, entendida esta como comunicación mediada (lo interpsicológico precede a lo intrapsicológico, según la conocida "ley genética general del desarrollo cultural”, de Vigotsky).

Es importante señalar que esta causación no es mecánica ni unilateral. Varios representantes de esta corriente (Baquero, 1996; Cubero \& Rubio, 
2005; Rogoff, 1993 ; Santigosa, 2005; Valsiner, 1991) resaltan el papel del dinamismo individual frente al entorno sociocultural.

Para la Teoría del AC, el enfoque sociocultural no solo es aplicable a la dimensión genética del desarrollo temprano (formación de la conciencia primaria), sino también a todo contexto vincular y comunicativo donde esté en juego el crecimiento psicológico personal a través de instancias de aprendizaje (apropiación cultural). El trabajo en colaboración es, sin duda, y de modo privilegiado, uno de estos contextos. En este caso, la relación con los otros no implica solo la confrontación de puntos de vista distintos, sino la posibilidad de construir una real intersubjetividad a partir de la convergencia de individualidades, donde lo colectivo es irreductible a lo individual. La significación de esta construcción intersubjetiva en colaboración reside en los importantes logros y progresos cognitivos individuales que se derivan.

En el enfoque neo-vygotskiano del aprendizaje colaborativo, el valor de la experiencia sociocomunicativa no radica solo en el acceso a una pluralidad de perspectivas, sino en los beneficios que implica la coordinación social en sí misma: el andamiaje y la ayuda mutua, la estimulación recíproca, la ampliación del campo de acción o de representación, la complementación de roles y el control intersujetos de los aportes y de la actividad.

Los representantes más reconocidos de esta línea son, sin duda, Cole, Valsiner, Rogoff, Perret-Clermont (en su segunda época), Wertsch y Cazden (todos participantes en la obra fundacional más emblemática de este grupo: "Perspectives on Socially Shared Cognition" [Resnick, J., Levine, J. \& Teasley, S., eds.], publicada por la APA en 1991). En castellano no se puede dejar de citar el número especial de Infancia y Aprendizaje, el № 27 28, editado en el 50 aniversario del fallecimiento de Vigotsky, que incluye tres capítulos que se refieren específicamente al aprendizaje entre pares: uno de Coll, otro de Forman-Cazden y otro de Webb. 


\section{La Teoría de la Cognición Distribuida.}

Esta corriente es altamente heterogénea y, por esto, no da lugar a una teoría en sentido estricto. Tampoco cada autor encuadrable en esta posición realiza una formulación teórica sistemática. En realidad, la intención del enfoque es descriptiva y pragmática, más que explicativa.

Esta heterogeneidad va desde una postura muy cercana al enfoque sociocultural (por ejemplo, Cole y Engeström) y a la cognición situada (Lave, Suchman), hasta una más cercana a los modelos sistémicos de procesamiento social cognitivo (Hutchins, Dillenbourg).

El concepto de cognición distribuida emerge como una postura crítica dentro de la psicología cognitiva y, más aún, de la ciencia cognitiva. La idea fundamental es que el procesamiento de información que se realiza a escala humana no es un fenómeno exclusivamente individual, mental o interno. La cognición humana está anclada en el contexto social y cultural en el que ocurre (en este sentido, se habla de cognición situada) y, por eso, el funcionamiento cognitivo no debe considerarse en términos de conciencia individual, sino "distribuido" en el entorno de herramientas y agentes sociales intervinientes. Esto implica que el grupo puede ser considerado una unidad de funcionamiento cognitivo, o sea, un sistema cognitivo. Pero este sistema incluye además, como elementos del sistema y no como mero contexto exterior, las tecnologías e instrumentos concurrentes. El entorno, dice Perkins (2001), "es verdaderamente una parte del pensamiento" (p. 136). Así, es lícito decir, por ejemplo, que un alumno piensa con y por medio de su cuaderno.

Siguiendo a este autor, es pertinente distinguir entre la cognición físicamente repartida (cuadernos, apuntes, carpetas, calculadoras, computadoras), la cognición socialmente distribuida (equipos o grupos de 
trabajo, organizaciones) y la cognición simbólicamente repartida (diagramas, mapas conceptuales, gráficos).

Algunos autores ponen el acento en la distribución social de la cognición (Hutchins, 1991; Dillenbourg \& Self, 1992; Minsky, 1986). A1 respecto dice Resnick (1991): "La metáfora de los sistemas cognitivos como sistemas sociales... hace a la comunidad de la ciencia cognitiva más abierta que una década atrás a la idea del conocimiento como distribuido a través de varios individuos cuyas interacciones determinan decisiones, juicios y solución de problemas" (p. 3).

Corresponde señalar que el concepto de cognición distribuida se desarrolló como una forma de abordar el estudio de la interacción hombrecomputadora (Hollan, Hutchins \& Kirsh, 1999; Dillenbourg et al., 1996). Este abordaje consiste en considerar la interacción usuario-sistema como un proceso socialmente distribuido. La noción de sistema cognitivo extendible más allá de lo puramente individual permite definir al grupo como un sistema de procesamiento, donde los individuos serían considerados agentes o componentes del mismo. Un sistema cognitivo deviene así una sociedad de agentes, independientemente de quienes sean estos (neuronas, individuos, subgrupos). Esta analogía entre sistemas de cognición individual y sistemas de cognición social despierta no pocas críticas (Nickerson, 2001), pero no cabe duda de su valor heurístico.

En este contexto, es entendible la relación de este enfoque con el del llamado "nuevo conexionismo". Nos referimos al Procesamiento Distribuido en Paralelo (PDP), y más específicamente al modelo computacional de Redes Neuronales, que es una alternativa útil para la comprensión de los fenómenos de procesamiento colectivo. La utilización de estos modelos permite, entre otras cosas, la simulación de sistemas cognitivos distribuidos, manipulando distintos grados de complejidad (Bruno, 1999). 


\section{Otras fuentes.}

Las fuentes teóricas mencionadas son la expresión del fuerte auge de la perspectiva constructivista y socioconstructivista en la psicología y la educación, que puso el énfasis no tanto en el resultado de la asociación cognitiva, sino en el proceso de negociación y construcción de significados compartidos. Pero también hay que tener en cuenta el fuerte desarrollo actual de las tecnologías de la información y la comunicación, las que habilitaron un sector propio de investigación: el aprendizaje colaborativo mediado por computadora ("Computer Supported Collaborative Learning", conocido por sus siglas CSCL), expresión más específica de la comunicación mediada por computadora (“Computer Mediated Communication”, o CMC). La fecundidad del mismo ha generado incluso serios trabajos dirigidos a ofrecer una síntesis de los aportes y una sistematización del campo (Dillenbourg, Baker, Blaye \& O’Malley, 1995; Dillenbourg \& Schneider, 1995; Rodríguez Illera, 2001; Valcke \& Martens, 2006; Weinberger et al., 2007; Beers et al., 2007).

En general, hay consenso en sostener que la aplicación de las actuales tecnologías comunicacionales a la educación implica el fortalecimiento de una concepción cooperativa del aprendizaje, o si se quiere, socioconstructivista (Gros Salvat, 2002; Kanselaar, Erkens, Jaspers \& Schijf, 2001; Kirschner, 2001; Koschmann, 1996).

No sería justo terminar esta enumeración de antecedentes en la temática sin una referencia a la corriente de Dinámica de Grupo, que en los años 60 tuvo fuerte desarrollo, sobre todo de la mano de la psicología dinámica (recuérdense los "grupos operativos", los "grupos-t" y los "grupos terapéuticos"). Pero también corresponde tener presente que la utilización acrítica y abusiva de las técnicas grupales en la educación en esos años causó un significativo desprestigio de estas, especialmente porque el énfasis en los aspectos afectivos y socio-relacionales hizo olvidar los aspectos 
específicamente cognitivos que son centrales en el aprendizaje escolar (cosa que no ocurre con la actual corriente del Aprendizaje Colaborativo). De todos modos, el trabajo con grupos, heredero de esa tradición psicosocial dinámica, nunca perdió vigencia en el campo organizacional, de trabajo social, de salud y de la educación comunitaria (García et al., 2008).

\section{Estrategias para el desarrollo de la colaboración.}

El auge contemporáneo de las corrientes socioconstructivistas en educación, y más específicamente del aprendizaje colaborativo, es a veces más retórico que real, dando lugar a posturas ingenuas, espontaneístas y abstractas de lo que implica trabajar cognitivamente en compañía. Se piensa que la condición social es un dato primario y natural del desarrollo humano, y que, por lo tanto, hay una disposición espontánea de los alumnos a la sociabilidad que está disponible. No se tiene en cuenta que el "habitus" de la individualidad está muy arraigado en las instituciones educativas, sobre todo en lo que hace al aprendizaje académico y a la apropiación de conocimientos. El actuar coordinadamente con otros, la creación de consensos, el aprovechamiento de los puntos de vista ajenos, son considerados buenas habilidades sociales que tienen que ver con el desarrollo de la sociabilidad y la convivencia, pero poco con la apropiación intelectual. El sujeto de aprendizaje (académico) es el individuo; cuesta entender que es posible plantearse la idea de un sujeto cognitivo colectivo.

Cuando se intenta romper la inercia de la enseñanza-aprendizaje individualista, se suele caer en la ingenuidad de que basta crear situaciones de conectividad horizontal entre los alumnos para que esto conduzca a un aprendizaje eficaz. Esta concepción es errónea. La colaboración sociocognitiva puede y debe ser desarrollada como una competencia en sí misma (Roselli, 1999b). El docente debe enseñar a trabajar eficazmente en un entorno colaborativo, y debe hacerlo utilizando estrategias específicas intencionalmente planificadas y muy pautadas. Precisamente, el trabajo 
propone un modelo integrado de estrategias que el docente puede utilizar en diferentes momentos del proceso de enseñanza. Estas estrategias se refieren a los diferentes aspectos (ejes) que normalmente están involucrados en todo proceso de enseñanza-aprendizaje de conocimientos: interacción con los otros, negociación y elaboración de consensos, organización de la actividad, apropiación de información, elaboración conceptual, redacción comunicativa. No son las únicas posibles; cada docente puede construir su propio repertorio a partir de estas referencias orientadoras. Es más, no todas deben introducirse abruptamente y en bloque. Lo deseable es una introducción gradual, sin perder de vista por esto que el objetivo final debería ser la implementación de un modelo didáctico colaborativo integral, y no quedarse en el uso de determinadas estrategias colaborativas sueltas y ocasionales. La idea de aprendizaje colaborativo implica una transformación general de la actitud didáctica, esto es, la modificación de los cimientos mismos del enseñar y del aprender. Es por esto que se prefiere hablar de estrategias para el desarrollo de la colaboración, y no meramente de técnicas (como se las nombra habitualmente en los ámbitos anglosajones). No hay que olvidar que, por definición, la estrategia es un constructo más amplio que el de técnica; las técnicas se seleccionan en función de las estrategias. Por sí solas, las técnicas son un barco sin guía.

\section{La noción de modelo didáctico colaborativo.}

Aunque ya se aludió anteriormente a la cuestión, no está de más reiterar el sentido que tiene el concepto de modelo en el contexto de este trabajo. Un modelo es, ante todo, integración de partes dentro de un todo coherente. Un modelo es una construcción conceptual cuyo fin es servir de guía y orientación de la acción. Es, ante todo, una entidad formal que ilumina la práctica, pero no es la práctica misma. Es por eso que no es una normativa rígida y cerrada, sino una guía lo suficientemente flexible como para poder operar en contextos didácticos diversos. Pero lo sustancial cuando se habla de modelo es la coherencia y unidad de concepción que debe tener. Esta es 
la diferencia esencial entre una didáctica basada en un modelo colaborativo integrado (o sea, una didáctica colaborativa auténtica) y una didáctica que solo emplea técnicas ocasionales de colaboración para dinamizar el aprendizaje o conseguir propósitos específicos en una fase de la enseñanza. En la literatura anglosajona existen abundantes referencias a esta aplicación pragmática de la colaboración (Barkley, Croos \& Major, 2007), pero la propuesta de este trabajo va más allá. Es una propuesta de cambio estructural del modelo didáctico, y no solo de técnicas procedimentales. Por eso se habla de estrategias, admitiendo que, si bien la enumeración de las propuestas que siguen incluye una dimensión técnica, no se agotan en esto, es decir, se integran dentro de una concepción que define orientaciones de acción, más que procedimientos (y solo eso) específicos.

En síntesis, todas las estrategias que constituyen el modelo propuesto tienen un leit-motiv, en el sentido de que apuntan a recuperar y desarrollar el vínculo sociocognitivo, la coordinación con los demás, la coparticipación en la construcción conceptual y el aprendizaje de la acción comunitaria. La implementación paulatina de dichas estrategias conduce finalmente a la concreción de un modelo didáctico colaborativo coherente y auténtico. En otras palabras, el modelo de estrategias presentado es la vía de entrada para el desarrollo de un modelo didáctico colaborativo.

El modelo que se presenta forma parte de un proyecto de investigación-acción que se está implementando actualmente en diversas cátedras universitarias de Argentina. La idea básica del proyecto es hacer una transformación gradual y paulatina del modelo didáctico de la enseñanza, pasando de uno fundamentalmente instructivo-individualista a otro integradamente colaborativo. En otras palabras, con la aplicación del modelo de estrategias propuesto se intenta avanzar hacia la implementación de un modelo didáctico con una identidad (teórica y práctica) enteramente colaborativa. La opción por el nivel universitario no es casual. En este nivel, el profesor cuenta con mayor margen de autonomía para efectuar cambios de 
fondo. Por otra parte, la colaboración en situaciones de aprendizaje requiere una dosis importante de autorregulación, sentido crítico y madurez de parte de los estudiantes, sobre todo para comprender el sentido del cambio de la intención didáctica que se pretende implementar, superar las resistencias y lograr la adhesión necesaria.

\section{Modelo de estrategias aplicables en la enseñanza universitaria.}

A continuación se presenta el modelo propuesto de estrategias de Aprendizaje Colaborativo. Las mismas están clasificadas según el aspecto sociocognitivo al que están dirigidas. Debe tenerse presente que todas estas estrategias están pensadas para ser aplicadas presencialmente, pero es posible adecuarlas para una aplicación en entornos virtuales, recurriendo a las diversas tecnologías comunicacionales. De hecho, hay importantes desarrollos de programas informáticos pensados específicamente para la colaboración sociocognitiva.

\section{Estrategias para el estímulo del diálogo, la escucha del otro y la evaluación recíproca.}

\section{Tormenta de ideas en cadena o rueda de ideas.}

Objetivo: Estímulo de la expresividad, la comunicación espontánea y la participación.

Descripción:

- Grupos de ocho sujetos.

- El docente propone un tema, de significación empírica, en relación con la materia.

- Cada sujeto, en ronda, escribe una breve frase y la coloca secuencialmente en una pizarra grupal.

- Se reitera la ronda tres veces.

- Se comparan colectivamente las pizarras de cada grupo. 


\section{Transmisión mediada de opiniones.}

Objetivo: Transmisión fidedigna de la escucha.

Descripción:

- Grupos de cuatro sujetos.

- Sobre un hecho o problema ofrecido por el docente, en cada tétrada, $\mathrm{S} 1$ emite una opinión a S2, y S2 hace lo mismo con S1. A su vez, S3 emite una opinión a S4, y S4 hace lo mismo con S3.

- Luego, se reitera la circulación de mensajes no-propios entre S1 y S3, y S2 y S4, y entre S1 y S2, y S3 y S4.

- Finalmente, cada sujeto escribe la opinión de los demás en función de lo que le fue transmitido.

- Evaluación conjunta en cada tétrada y evaluación grupo-clase.

\section{Evaluación recíproca por pareja.}

Objetivo: Estímulo de la evaluación del otro.

Descripción:

- En pareja.

- Cada sujeto corrige y evalúa la/s respuesta/s escrita/s (de un ejercicio) de su alter, de acuerdo con su criterio.

- En función del modelo correcto presentado por el docente, cada uno es corregido por un integrante de otra pareja o por ambos.

\section{Comisión evaluadora.}

Objetivo: Promover criterios colectivos de evaluación y estímulo del control intersubjetivo.

Descripción:

- Grupos de cuatro sujetos.

- La producción de cada sujeto de un grupo es evaluada por un comité de pares (miembros de otro grupo), quienes emiten un dictamen escrito. 
- Los cuatro sujetos evaluados comparan y discuten sus dictámenes.

- Cada sujeto contraargumenta o responde a la evaluación recibida.

- El docente explicita su criterio experto en general, dirimiendo los conflictos particulares.

\section{Simetría de la participación.}

Alternancia obligatoria de la participación.

Objetivo: Sostenimiento de la equidad en la participación.

Descripción:

- Grupos de cuatro sujetos.

- Se pauta la participación de la performance grupal, de modo que cada sujeto, siguiendo una secuencia obligatoria, debe hacer un aporte o resolver un ítem.

- Los aportes se registran en una pizarra grupal.

- La técnica puede complementarse con la evaluación de cada aporte, hecha también en un orden secuencial obligatorio.

Cuota de participación.

Objetivo: Dosificación de la participación equitativa.

Descripción:

- Grupos de cuatro sujetos.

- Cada sujeto dispone de una determinada cuota de participaciones, que puede administrar libremente, pero que al finalizar la tarea debe haberse usado.

- Si por contingencias del desarrollo de la tarea algún sujeto no completara el uso de su cuota, dispondrá de un crédito obligatorio para la próxima tarea. 


\section{Estrategias de colaboración para la negociación y la creación de consenso.}

\section{Debate crítico pautado.}

Objetivo: Estímulo del intercambio y de la confrontación de ideas.

Descripción:

- Grupos de cuatro sujetos.

- En relación con un problema dado, cada uno prepara su propia argumentación y, a su turno, la presenta al grupo.

- Los otros miembros apoyan o refutan.

- El sujeto ejerce su derecho a réplica.

- Al final, entre todos, se hace un ordenamiento o clasificación de las cuatro posiciones.

\section{Debate crítico con alternancia de posiciones.}

Objetivo: Desarrollo de la capacidad de ponerse en el lugar del otro.

Descripción:

- En pareja.

- En un tema de debate (ej.: experimentación con células madre), cada sujeto del grupo-clase argumenta o defiende espontáneamente una posición (oralmente o por escrito).

- En función de los productos, el docente asocia sujetos de opiniones diferentes y solicita un cambio de roles, debiendo cada sujeto buscar argumentos contrarios a su opinión espontánea.

- El alter comenta y amplía la argumentación.

Variante: Las parejas se constituyen por afinidad de opinión, debiendo coordinar una argumentación común. 


\section{Ponderación colectiva de alternativas en una situación de toma de decisión.}

Objetivo: Desarrollo de la capacidad analítica grupal.

Descripción:

- Grupos de cuatro sujetos.

- Frente a una situación de toma de decisión ofrecida por el docente, listar todas las alternativas posibles, ordenándolas jerárquicamente.

- Seleccionar las primeras del ranking y detallar los pro y contra de cada una.

\section{Mesa redonda con moderador y elaboración de conclusiones de consenso.}

Objetivo: Capacidad para acercar posiciones y crear consenso.

Descripción:

- Grupos de cuatro sujetos más uno adicional.

- En un tema ofrecido por el docente, cada sujeto hace una breve disertación frente al grupo-clase.

- Un moderador acerca posiciones y gestiona la elaboración de conclusiones consensuadas, que él escribe y lee al grupo para su aprobación.

- Cada sujeto deberá ser moderador en otros grupos.

\section{Conferencia comentada.}

Objetivo: Estímulo de la crítica y de la ampliación de perspectivas.

Descripción:

- Grupos de cuatro sujetos.

- Un sujeto del grupo prepara y realiza una disertación ante el grupoclase. 
- Otros dos sujetos del grupo, habiendo leído previamente la disertación, realizan un comentario crítico, que también presentan ante la clase.

- Un cuarto sujeto actúa de moderador y promotor de conclusiones de consenso.

- En sucesivas oportunidades, los roles deben cambiarse.

- Los otros grupos discuten brevemente, y un representante de cada uno realiza un comentario o reflexión complementaria.

\section{Grupo de discusión con observadores ("pecera").}

Objetivo: Estímulo de la metacognición social.

Descripción:

- Grupos de cuatro discutidores y cuatro observadores.

- Los cuatro miembros discutidores consideran el tema propuesto.

- Los cuatro miembros de otro grupo actúan de observadores.

- Después de la discusión, los cuatro observadores realizan un análisis del intercambio sociocognitivo.

- Posteriormente, hay rotación funcional entre ambos grupos.

\section{Estrategias de organización de la actividad.}

\section{Realización de mini-investigaciones teóricas o bibliográficas.}

Objetivo: Desarrollo de la capacidad de organizarse y organizar la realización conjunta de una tarea de una cierta complejidad. Sistematización de la actividad.

Descripción:

- Grupos de cuatro sujetos.

- En base a un tema propuesto por el docente, dividir la tarea según estas funciones, distribuyendo las mismas entre los miembros: a) búsqueda en las bases bibliográficas de Internet y páginas web, b) 
búsqueda en las librerías y bibliotecas, c) entrevistas a especialistas y expertos, d) consulta a expertos por correo electrónico.

- En base a los informes parciales redactados por los miembros responsables, elaborar un informe único, el que será presentado oralmente al grupo-clase.

\section{Realización de mini-investigaciones empíricas exploratorias.}

Objetivo: Los mismos de la técnica anterior, solo que, en este caso, la tarea es de mayor complejidad.

Descripción:

- Grupos de cuatro sujetos.

- En base a un tema aprobado por el docente, dividir la tarea según estas funciones, distribuyendo las mismas entre los miembros:

- Información de fuentes secundarias y terciarias: a) búsqueda bibliográfica, b) entrevistas a especialistas y testigos calificados, c) búsqueda de datos estadísticos y material documental.

- Información de fuentes primarias: a) observación de campo, b) aplicación de cuestionarios, c) realización de entrevistas.

- Procesamiento y ensamblaje de la información.

- Elaboración de un informe único, el que será presentado oralmente al grupo-clase.

- Todos los miembros deben participar (aunque sea parcialmente en virtud de la división de funciones) en las actividades de los puntos A, B, C y D.

\section{Estrategias de estudio y apropiación de la información bibliográfica.}

Enseñanza recíproca ("rompecabezas" o "jigsaw classroom").

Objetivo: Desarrollo de la función tutorial.

Descripción: 
- Grupos de cuatro sujetos.

- Uno de los miembros (función rotativa) es convocado por el docente, creando un grupo ad-hoc con todos los seleccionados de cada grupo.

- El docente trabaja intensivamente con ellos una pieza bibliográfica.

- Posteriormente, cada estudiante vuelve a su grupo y enseña dicho material bibliográfico a sus tres otros pares, a través de la lectura dirigida en situación.

- El par-tutor responde todas las consultas y se asegura de una correcta comprensión por parte de sus pares-alumnos.

- Finalmente, con la tutoría del par-experto, los tres restantes realizan una síntesis conceptual respondiendo a una serie de preguntas-guía.

- El rol de par-tutor va rotando en función de la secuencia bibliográfica.

\section{Estudio domiciliario.}

Objetivo: Sistematización en clase de la lectura bibliográfica domiciliaria. Descripción:

- Grupos de cuatro sujetos.

- El docente distribuye con anticipación la pieza bibliográfica y una guía de lectura, requiriendo su lectura domiciliaria individual.

- El día fijado, el docente destina un cierto tiempo para la relectura individual, implementando luego una fase de revisión por pareja, con la ayuda de la guía de lectura.

- Finalmente se reúnen las dos parejas de cada grupo y realizan una síntesis conceptual, respondiendo a una serie de preguntas-guía proporcionadas por el docente.

\section{Desarrollo grupal de la comprensión lectora.}

Objetivo: Desarrollar la comprensión lectora a través del intercambio y el consenso intersubjetivo.

Descripción:

- Grupos de cuatro sujetos. 
- De un texto conceptual-expositivo, producir grupalmente:

- Un resumen breve (extensión variable).

- Título temático (alternativo si ya lo tiene).

- Reconocer las ideas centrales, diferenciándolas de las periféricas.

- Reconocer la estructura del mismo (partes, subtemas, niveles de análisis, secuencia argumentativa).

\section{Estrategias de elaboración conceptual.}

\section{Comparación en pareja de apuntes de clase y elaboración conjunta de una versión mejorada.}

Objetivo: Mejoramiento de la comprensión retrospectiva a través del chequeo interpersonal y la comparación recíproca de los registros de clase. Estímulo de la descentración cognitiva.

Descripción:

- En pareja.

- Se solicita explícitamente que todos tomen apuntes de la clase.

- Luego, cada alumno se asocia con un alter, realizando entre los dos una comparación entre ambos apuntes.

- Como resultado de la misma, cada pareja crea una versión mejorada.

- Algunas parejas presentan sus conclusiones ante el grupo-clase con los comentarios del docente.

\section{Integración temática (bibliográfica) por pareja.}

Objetivo: Desarrollo de la competencia de integración conceptual.

Descripción:

- En pareja y grupos de cuatro sujetos.

- Cada pareja es responsable de realizar la integración temática requerida (sobre la base de varias fuentes de información) con la ayuda de una guía, debiendo producir un escrito al respecto. 
- Posteriormente, cada pareja se reúne con otra para evaluar conjuntamente ambas producciones, elaborando un nuevo escrito.

- Este es corregido por el docente y devuelto para la consideración grupal.

\section{Circulación de preguntas y respuestas.}

Objetivo: Desarrollar la capacidad de formular preguntas pertinentes y medulares en relación con un contenido temático.

Descripción:

- Grupos de cuatro sujetos.

- Cada grupo propone las preguntas esenciales que habría que hacer a un alumno para saber el grado de dominio de un tema.

- Las preguntas de cada grupo son contestadas por otro grupo (orden azaroso).

- Cada grupo autor de las preguntas evalúa las respuestas producidas por el otro grupo.

- Todos los grupos son emisores de preguntas y de respuestas.

- Finalmente, el docente evalúa las evaluaciones grupales.

\section{Elaboración grupal de organizadores conceptuales.}

Objetivo: Estimular la creatividad y poder de síntesis conceptual.

Descripción:

- Grupos de cuatro sujetos.

- A posteriori de un desarrollo temático, cada grupo elabora interactivamente:

- Esquemas temáticos y cuadros sinópticos.

- Mapas conceptuales.

- Cada grupo compara su producción con la de otro grupo. 


\section{Estrategias de escritura colectiva.}

\section{División de funciones en la redacción de un informe colectivo.}

Objetivo: Sistematizar la compleja actividad que significa una redacción colectiva.

Descripción:

- Grupos de cuatro sujetos.

- Acordar la estructura y los puntos, ideas o ítems conceptuales que tendrá el informe, haciendo un listado abreviado de los mismos.

- Dividir las funciones para la redacción: un sujeto recuperará la idea (que puede volver a explicitarse entre todos), otro la dictará y otro la escribirá. El cuarto sujeto monitoreará el proceso.

- El texto final será leído y corregido por todos.

- Según el tipo de información, los roles pueden alternarse o no.

\section{Compatibilización de textos individuales para producir un texto colectivo.}

Objetivo: Desarrollar la capacidad de análisis y síntesis en la comparación de textos.

Descripción:

- En pareja.

- Acordar la estructura y los puntos, ideas o ítems conceptuales que tendrá el informe, haciendo un listado de los mismos.

- Cada sujeto redacta su texto propio.

- Se comparan en pareja los escritos individuales y se produce un nuevo texto consensuado. Puede tomarse uno de los textos como base de la redacción.

\section{Conclusiones}

La Teoría del Aprendizaje Colaborativo es la expresión más representativa del socioconstructivismo educativo. En realidad, no es una teoría unitaria, 
sino un conjunto de líneas teóricas que resaltan el valor constructivo de la interacción sociocognitiva y de la coordinación entre aprendices. Tres de estas corrientes merecen una referencia especial por ser las fuentes básicas de la Teoría del Aprendizaje Colaborativo: el socioconstructivismo neopiagetiano o teoría del conflicto sociocognitivo, el enfoque neo-vygotskiano de la intersubjetividad y el modelo de la cognición distribuida o pensamiento en red. Estas tres corrientes pueden situarse en un eje "individual-grupal", según que el énfasis se ponga en el individuo en interacción o en lo colectivo como tal. El orden en el que han sido nombradas define la respectiva ubicación en el eje.

La colaboración sociocognitiva requiere aprendizaje; esto es, puede y debe ser enseñada. El docente es el responsable de que los alumnos aprendan a colaborar eficazmente entre ellos, transformando la clase en una comunidad de enseñanza-aprendizaje. Para ello dispone de un variado dispositivo de estrategias que puede implementar en diferentes instancias, en función de distintos tipos de tarea (Roselli, 2011). Lo importante es entender que no se trata solo de recursos pedagógicos sueltos de formación para la colaboración, y menos de meras técnicas de animación grupal; se trata de una propuesta de un nuevo modelo didáctico que aproveche académicamente, de modo eficaz, la sociabilidad natural del contexto institucional de enseñanza, básicamente colectivo. Este modelo didáctico incluye el trabajo en equipo, pero no es solo eso. El encuadre didáctico colaborativo va más allá del simple colectivismo (hacer todo en grupo); incluye y mantiene las instancias individuales, solo que conecta el propio aprendizaje con el de los demás.

El modelo ofrecido en este trabajo no es el único posible; constituye sólo una referencia a partir de la cual cada docente construye el suyo (en función de la episteme, de su impronta individual, de las características de sus alumnos y del marco institucional).

Lo más enriquecedor y motivante es que el profesor se lo plantee como una investigación-acción de innovación pedagógica, involucrando 
participativamente al personal de cátedra y a los alumnos (Roselli, 2008). En este marco, puede incluso implementar condiciones de control (comparativas) no-colaborativas. También se pueden implementar instancias comparativas entre formas de trabajo grupal no-pautadas y pautadas por el docente de antemano, como una forma de asegurar un encuadre organizativo eficaz, cuya ausencia es lo que comúnmente dificulta la coordinación colectiva.

Otro problema no menor son las resistencias al cambio, sobre todo las encubiertas. Estas pueden provenir del o de los propios docentes involucrados declarativamente en la experiencia didáctica colaborativa, como así también de los alumnos. Los encuadres colaborativos entusiasman a ambos por la impronta innovadora que suponen y porque el rescate de la sociabilidad crea un mejor clima de trabajo, pero suponen nuevas y muy rigurosas exigencias de trabajo y actuación (preparación de materiales, cumplimiento de las tareas encomendadas, puntualidad, adecuación individual al funcionamiento colectivo), que no todos llegan a asumir en los hechos. Tampoco las regulaciones institucionales suelen ayudar mucho. El trasfondo de estas resistencias es, sin duda, la cultura individualista que, más allá de las declaraciones, impregna los ámbitos institucionales educativos. Es por esto que la implementación de estrategias colaborativas debe ser gradual, consensuada y acompañada de una permanente reflexión crítica de los logros. 


\section{Referencias}

Baquero, R. (1996). Vigotsky y el aprendizaje escolar. Buenos Aires: Aiqué.

Barkley, E. F., Croos, P., \& Major, C. H. (2007). Técnicas de aprendizaje colaborativo. Madrid: Morata.

Beers, P. J., Boshuizen, H. P., Kirschner, P. A., \& Gijselaers, W. H. (2007). The analysis of negotiation of common ground in CSCL. Learning and Instruction, 17, 427-435.

Bruffee, K. A. (1993). Collaborative Learning: Higher education, interdependence, and the authority of knowledge. Baltimore, MD: Johns Hopkins University Press.

Bruno, M. (1999). La cognición socialmente distribuida como forma particular de procesamiento distribuido en paralelo. Revista IRICE, 13, 135-143.

Coll, C. (1991). Aprendizaje escolar y construcción del conocimiento. Buenos Aires: Paidós.

Cubero, M., \& Rubio, D. (2005). Psicología histórico-cultural y naturaleza del psiquismo. En M. Cubero y J. Ramírez (comps.), Vigotsky en la Psicología Contemporánea. Buenos Aires: Miño y Dávila.

Dillenbourg, P., \& Self, J. (1992). A computational approach to socially distributed cognition. European Journal of Psychology of Education, 7, 353-372.

Dillenbourg, P., Baker, M., Blaye, A., \& O'Malley, C. (1995). The evolution of research on collaborative learning. In P. Reimann, \& H. Spada, Learning in Humans and Machines. Towards an Interdisciplinary Learning Science (pp. 189-211). London: Pergamon.

Dillenbourg, P., \& Schneider, D. (1995). Collaborative learning and the Internet. TECFA, University of Geneve. Recuperado de http://tecfa. unige.ch/tecfa/research/CMC/colla/iccai95_1.html

Dillenbourg, P., \& Self, J. (1992). A computational approach to socially distributed cognition. European Journal of Psychology of Education, 7(4), 353-371. 
Dillenbourg, P., Baker, M., Blaye, A., \& O’Malley, C. (1996). The Evolution of Research on Collaborative Learning. In P. Reimann, \& H. Spada (Eds.), Learning in Humans and Machines. Towards an Interdisciplinary Learning Science (pp. 189-211). London: Pergamon.

Dillenbourg, P. (1999). What do you mean by collaborative learning? In P. Dillenbourg (Ed.), Collaborative-learning: Cognitive and Computational Approaches. Amsterdam: Elsevie Science.

Exley, K., \& Dennick, R. (2007). Enseñanza en pequeños grupos en educación superior. Tutorías, seminarios y otros agrupamientos. Madrid: Narcea.

García, D., Robles, C., Rojas, V., \& Torrelli, A. (2008). El trabajo en grupos. Aportes teóricos e instrumentales. Buenos Aires: Espacio.

Gros Salvat, B. (2002). Constructivismo y diseños de entornos virtuales [Constructivism and the design of virtual environments]. Revista de Educación, 328, 225-247.

Hollan, J., Hutchins, E., \& Kirsh, D. (1999). Distributed cognition: a new foundation for human-computer interaction research. Recuperado de www.HCI.UCSD.EDU.

Huertas, J. A. (2001). La interacción en el aula. Buenos Aires: Aiqué.

Hutchins, E. (1991). The social organization of distributed cognition. In L. Resnick, J. Levine, \& S. Teasley (Ed.), Perspectives on Socially Shared Cognition. Washington: American Psychological Association, pp. 283307.

Johnson, D., \& Johnson, R. (1999). Aprender juntos y solos. Buenos Aires: Aiqué.

Kanselaar, G., Erkens, G., Jaspers, J., \& Schijf, H. (2001). Computer supported collaborative learning (Essay review). Teaching and Teacher Education, 17, 123-129.

Kirschner, P. (2001). Using integrated electronic environments for collaborative teaching/learning. Research Dialogue in Learning and Instruction, 2, 1-9.

Koschmann, T. (Ed.). (1996). CSCL: Theory and practice of an emerging paradigm. Mahwah, NJ: Lawrence Erlbaum Associates. 
Lewis, R. (2003). Pourquoi apprendre à collaborer. En C. Bernadette, \& D. Peraya (Eds.). Technologie et Innovation en Pédagogie. Dispositifs Innovants de Formation Pour l'Enseignement Supérieur. Éditions De Boeck Université: Bruxelles, Cap. 10, pp. 137-139.

Melero Zabal, M., \& Fernández Berrocal, P. (1995). El aprendizaje entre iguales: El estado de la cuestión en Estados Unidos [Peer learning: the state of the art in USA]. En P. Fernández y M. Melero (Eds.), $L a$ interacción social en contextos educativos (Cap. 2, pp. 35-98). Madrid: Siglo XXI.

Minsky, M. (1986). La Sociedad de la Mente. Buenos Aires: Ediciones Galápago.

Nickerson, R. (2001). Algunas reflexiones acerca de la distribución de la cognición. En G. Salomón (Ed.), Cogniciones Distribuidas. Buenos Aires: Amorrortu.

Panitz, T. (1997). Collaborative versus cooperative learning -a comparison of the two concepts which Hill help us understand the underlying nature of interactive learning. Cooperative Learning and College Teaching [artículo en línea], 8(2). Recuperado de www.ufv.br/dpe/edu660/textos/ t14_aprend_colab_def.rtf

Perkins, D. (2001). La Escuela Inteligente. Barcelona: Gedisa.

Quiamzade, A., Mugny, G., \& Butera, F. (2013). Psychologie Sociale de la Connaissance. Grenoble: Presses Universitaires de Grenoble.

Resnick, L. (1991). Shared cognition: thinking as a social practice. In L. Resnick, J. Levine, \& S. Teasley (Eds.), Perspectives on Socially Shared Cognition (pp. 1-20). Washington, DC: American Psychological Association.

Rodriguez Barreiro, L., Fernández, R., Escudero, T., \& Sabirón, F. (2000). La investigación sobre el aprendizaje colaborativo: Enfoques, métodos y resultados [Collaborative learning research: approaches, methods and results]. Anuario de Pedagogía, 2, 305-338.

Rodriguez Illera, J. (2001). Aprendizaje colaborativo en entornos virtuales [Collaborative learning in virtual environments]. Anuario de Psicología, 32(2), 63-75. 
Rogoff, B. (1993). Children's guided participation and participatory appropiation in sociocultural activity. In R. Wozniak, \& K. Fisher (Eds.), Development in Context. Hillsdale, NJ: LEA, pp. 121-153.

Roselli, N. (1999a). La Construcción Sociocognitiva entre Iguales. Rosario: IRICE.

Roselli, N. (1999b). El mejoramiento de la interacción sociocognitiva mediante el desarrollo experimental de la cooperación auténtica. Interdisciplinaria, 16(2), 123-151.

Roselli, N. (2007). El aprendizaje colaborativo: fundamentos teóricos y conclusiones prácticas derivadas de la investigación. En M. C. Richaud y M. S. Ison, Avances en Investigación en Ciencias del Comportamiento en Argentina. Capítulo 18, Tomo I, pp. 481-498. Editorial de la Universidad del Aconcagua: Mendoza.

Roselli, N. (2008). La disyuntiva individual-grupal. Comparación entre dos modalidades alternativas de enseñanza en la universidad. Revista Ciencia, Docencia, Tecnología, 36, 87-120.

Roselli, N. (2011). Diferencias en el proceso de construcción colaborativa a través del chat según el tipo de tarea. Revista de Psicología, 29(1), 3-36.

Santigosa, A. (2005). La noción de interiorización desde una visión cultural del desarrollo. En M. Cubero y J. Ramírez (comps.), Vigotsky en la Psicología Contemporánea. Buenos Aires: Miño y Dávila.

Slavin, R. (1999). Aprendizaje Cooperativo. Buenos Aires: Aiqué.

Strijbos, J., \& Fischer, F. (2007). Methodological challenges for collaborative Learning Research, 17(4), 389-464.

Valcke, M., \& Martens, R. (2006). The problem arena of researching computer supported collaborative learning: Introduction to the especial section. Computer \& Education, 46, 1-5.

Valsiner, J. (1991). Building theoretical bridges over a lagoon of everyday events. A review of apprenticeship in thinking: cognitive development in social context by Barbara Rogoff. Human Development, 34, 307-315.

Weinberger, A., Stegmann, K., \& Fischer, F. (2007). Knowledge convergence in collaborative learning: Concepts and assessment. Learning and Instruction, 17, 416-426. 